\title{
Bleeding Complications and Clinical Safety of Endoscopic Retrograde Cholangiopancreatography in Patients with Liver Cirrhosis
}

\author{
Ji Yeon Kim*, Hee Seung Lee*, Moon Jae Chung, Jeong Youp Park, \\ Seung Woo Park, Si Young Song, and Seungmin Bang \\ Department of Internal Medicine, Institute of Gastroenterology, Yonsei University College of Medicine, Seoul, Korea.
}

\begin{abstract}
Purpose: Patients with liver cirrhosis are considered to be at risk for additional adverse events during endoscopic retrograde cholangiopancreatography (ERCP). The present study was designed as a propensity-score matched analysis to investigate whether cirrhotic liver increases the risk of bleeding complications in patients undergoing ERCP.

Materials and Methods: In total, 8554 patients who underwent ERCP from January 2005 to December 2015 were retrospectively analyzed. To adjust for the imbalance between patients with and those without liver cirrhosis, 1:3 propensity score matching was performed according to age and sex.

Results: Liver cirrhosis was identified in 264 (3.1\%) patients. After propensity score matching, a total of 768 patients were included in each of the cirrhotic $(\mathrm{n}=192)$ and non-cirrhotic groups $(\mathrm{n}=576)$. Post-procedure bleeding $(10.9 \%$ vs. $4.7 \%, p=0.003)$ was more frequently observed in patients with liver cirrhosis than in those without. In multivariate analyses, liver cirrhosis was identified as an independent risk factor associated with post-ERCP bleeding ( $p=0.003)$ after further adjustment for prothrombin time, antiplatelet/coagulant, duration of ERCP, and stent insertion. Child-Pugh (CP) class C was found to be associated with an increased incidence of post-ERCP bleeding in patients with cirrhosis (odds ratio 6.144, 95\% confidence interval 1.320-28.606; $p=0.021$ ).

Conclusion: The incidence of post-ERCP bleeding in patients with liver cirrhosis was higher than that in patients without liver cirrhosis. In particular, CP class C cirrhosis was significantly associated with post-ERCP bleeding.
\end{abstract}

Key Words: Liver cirrhosis, endoscopic retrograde cholangiopancreatography, hemorrhage

\section{INTRODUCTION}

Endoscopic retrograde cholangiopancreatography (ERCP) has become the first-choice treatment for common bile duct (CBD) disorders and periampullary disease. ${ }^{1,2}$ Compared with

Received: October 29, 2018 Revised: March 5, 2019

Accepted: March 11, 2019

Corresponding author: Seungmin Bang, MD, PhD, Division of Gastroenterology, Department of Internal Medicine, Yonsei University College of Medicine, 50-1 Yonsei-ro, Seodaemun-gu, Seoul 03722, Korea.

Tel: 82-2-2228-1957, Fax: 82-2-2227-7900, E-mail: bang7028@yuhs.ac

* Ji Yeon Kim and Hee Seung Lee contributed equally to this work.

- The authors have no potential conflicts of interest to disclose.

(C) Copyright: Yonsei University College of Medicine 2019

This is an Open Access article distributed under the terms of the Creative Commons Attribution Non-Commercial License (https://creativecommons.org/licenses/ by-nc/4.0) which permits unrestricted non-commercial use, distribution, and reproduction in any medium, provided the original work is properly cited. other endoscopic examinations, ERCP carries a higher potential for complications, ranging from minor events with prompt resolution, such as mild pancreatitis, to major life-threatening risks, such as hemorrhage and severe pancreatitis..$^{3-7}$ In previous studies, the risk factors for bleeding as a result of ERCP are coagulopathy, liver cirrhosis, renal failure, sphincterotomy, and papillectomy. ${ }^{8-10}$ In particular, patients with liver cirrhosis have been considered to be at high risk for ERCP-related bleeding. ${ }^{11,12}$ Cirrhosis-associated coagulopathy, liver dysfunction, and gastroesophageal varices may increase the risk of procedure-related bleeding in these patients. ${ }^{4}$

To date, several studies have analyzed the risk of ERCP in patients with liver cirrhosis. ${ }^{11,13-15}$ However, previous studies reported limited sample sizes, ${ }^{12,15,16}$ small portions of therapeutic ERCPs ${ }^{14}$ limited information about the type of procedure or degree of cirrhosis, and substantial heterogeneity in the comparison group. ${ }^{11,12}$ Furthermore, the safety of ERCP in liver cir- 
rhosis is still controversial because of limited data. ${ }^{11,14}$ Adler, et al. ${ }^{14}$ reported that therapeutic ERCP is safe and effective in patients with liver cirrhosis. Meanwhile, however, Inamdar, et al. ${ }^{11}$ showed that the frequency of ERCP-related bleeding was statistically significant in patients with decompensated liver cirrhosis. Endoscopic sphincterotomy (EST) or balloon dilatation, which means cutting or dilatation of the sphincter muscle that lies at the juncture of the intestine with the bile ducts, is an essential technique in patients who receive ERCP. ${ }^{16-18}$ Prior to those procedures in cirrhotic patients, recognition and understanding of the potential complications of ERCP are important for appropriate patient selection for performing ERCP.

Here, we aimed to investigate the safety of ERCP in patients with liver cirrhosis, focusing on bleeding complications. For the evaluation of bleeding complications of ERCP in patients with cirrhosis, we performed propensity-score matched analysis using a retrospective cohort data.

\section{MATERIALS AND METHODS}

\section{Patients}

A total of 8554 patients had undergone ERCP at an academic tertiary hospital in South Korea between January 2005 and December 2015. Among them, 555 patients were excluded according to the following exclusion criteria: ERCP for bile duct anastomosis site stricture after liver transplantation in the cirrhotic group $(n=129)$; failed cannulation during the procedure $(n=$ 269); only cholangiography without insertion of a guidewire during the procedure $(n=93)$; and papillectomy $(n=64)$. Finally, 264 patients with cirrhosis and 7735 patients without cirrhosis remained.

The demographic, clinical, and laboratory data of the study population were collected from medical records. Medical record data, including sex, age at the time of procedure, etiology of cirrhosis, cardiovascular disease, medication related to bleeding tendency, pre-procedure laboratory values, Child-Pugh (CP) scores at the time of the procedure, ERCP indications, procedural details, and procedure-related adverse events, were reviewed. Liver cirrhosis was diagnosed according to laboratory values, liver biopsies, and the results of imaging studies. If liver histologic information was not available, clinically diagnosed liver cirrhosis was based on following criteria: 1) platelet count of $<100000 / \mu \mathrm{L}$ and ultrasonographic findings suggestive of cirrhosis, including a blunted, nodular liver edge accompanied by splenomegaly (>12 cm); 2) esophageal or gastric varices; or 3) overt complications of liver cirrhosis, including ascites, variceal bleeding, and hepatic encephalopathy. ${ }^{19}$ The severity of liver cirrhosis was classified according to CP class. This study was performed in accordance with ethical guidelines of the 1975 Declaration of Helsinki and approved by the Institutional Review Board of Severance Hospital (IRB number: 4-2016-1090).

\section{Propensity score matching}

Propensity score matching was introduced to reduce patient selection bias and the potential confounding biases due to differences between the two patient groups. A biostatistician in the Medical Research Collaborating Center matched the group of the patients with liver cirrhosis to the group of those without liver cirrhosis at a 1:3 ratio using the calculated propensity score by optimal matching methods. To eliminate bias caused by different baseline characteristics, we calculated propensity score based on age and sex. After optimal matching, we compared the baseline characteristics. Finally, 768 patients were matched, including 192 patients in the cirrhotic group and 576 patients in the non-cirrhotic group (Fig. 1).

\section{Endoscopic procedure}

Patients were sedated with intravenous anesthesia (midazolam and/or propofol), along with continuous monitoring of blood pressure, heart rate, and oxygen saturation. All patients received intravenous cimetropium bromide for inhibiting duodenal spasm. A side-viewing duodenal scope (TJF-260; Olympus, Tokyo, Japan) was used for ERCP. After the papilla had been examined with the endoscope, selective cannulation of either the CBD or the ventral pancreatic duct was performed. Once the chosen duct was selectively cannulated, either a cholangiogram or a pancreatogram was obtained fluoroscopically with injection of contrast medium into the duct. Four hours after the ERCP and at 5 a.m. of the next day of the procedure, blood chemistry, including serum amylase, lipase, and total bilirubin level; chest radiography; and simple abdominal radiography were performed for evaluating post-ERCP pancreatitis (PEP), cholangitis, and bowel perforation. To identify post-ERCP bleeding, hemoglobin level and bleeding history were checked. Most of the patients taking medications related to bleeding tendency (such as aspirin, warfarin, clopidogrel,

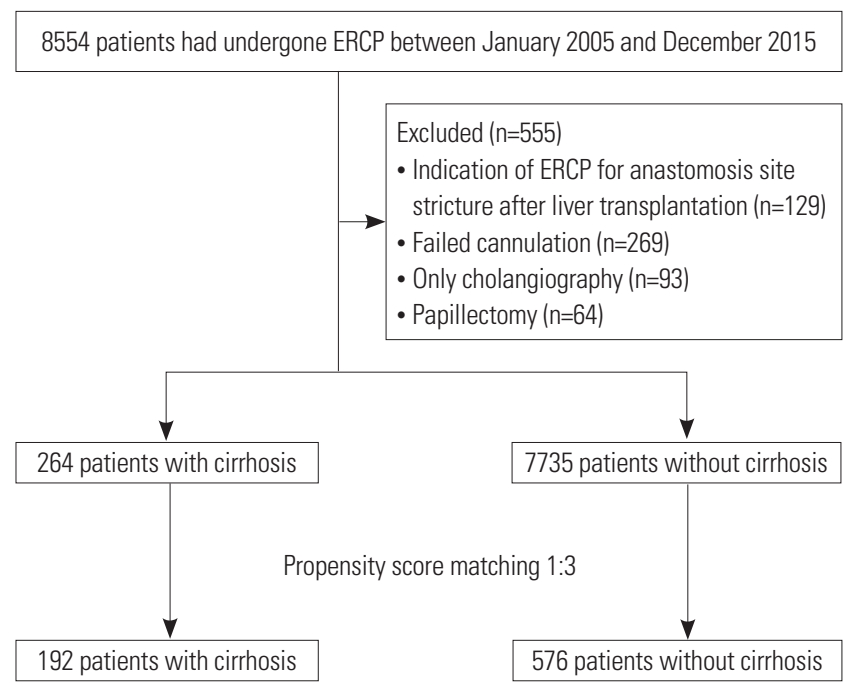

Fig. 1. Flow diagram of patient selection. ERCP, endoscopic retrograde cholangiopancreatography. 
or a new oral anticoagulant) needed to stop the medications for at least until 3 days before the procedure.

\section{Definition and management of complications}

Post-sphincterotomy bleeding was defined as immediate bleeding requiring endoscopic or other intervention within 24 $\mathrm{hr}$ or delayed bleeding recognized on the basis of clinical evidence (such as melena, hematochezia, and hematemesis), with a decrease in hemoglobin level $>2 \mathrm{~g} / \mathrm{dL}$ or the need for blood transfusion within 10 days after ERCP. ${ }^{4}$ Clinically significant bleeding and its severity were classified as follows: mild bleeding was defined as overt bleeding with a decrease of hemoglobin level $<3 \mathrm{~g} / \mathrm{dL}$, without the need for transfusion; moderate bleeding was defined as blood transfusion of 4 units or less without the need for angiographic intervention or surgery; and severe bleeding was defined as blood transfusion of 5 units or more or the need for angiographic or surgical intervention. ${ }^{20}$

\section{Statistical analysis}

Statistical analyses were performed using SPSS software (version 20.0; IBM Corp., Armonk, NY, USA). Associations involving parametric data were assessed using Student's t-test. Chisquare $\left(\chi^{2}\right)$ test was used to compare categorical data, and Student's t-test was used to compare continuous data. Statistical analysis was performed with the Jonckheere-Terpstra test to determine whether there was a statistically significant trend among independent samples. All two-tailed $p$ values of $<0.05$ were considered statistically significant.

\section{RESULTS}

\section{Patients}

After propensity score matching, a total of 192 patients with cirrhosis (age 59.4 \pm 9.5 years) were included in the analysis. The etiologies of cirrhosis were as follows: hepatitis B $(n=106$, $59.2 \%)$, hepatitis $\mathrm{C}(\mathrm{n}=21,11.7 \%)$, and alcoholic ( $\mathrm{n}=28,15.6 \%)$. Among 192 patients with liver cirrhosis, 92 (47.9\%) had CP classification A, 55 (28.6\%) had CP classification B, and 45 (23.4\%) had CP classification C. The patient demographic and procedure characteristics are summarized in Table 1. The indications for ERCP were as follows: CBD stone with or without acute cholangitis ( $\mathrm{n}=296,38.5 \%$ ), bile duct obstruction $(\mathrm{n}=335,43.6 \%)$, and pancreatitis $(\mathrm{n}=32,4.2 \%)$.

\section{ERCP-related bleeding complications}

A total of 48 patients had post-ERCP bleeding among the enrolled patients, and there was a statistically significantly higher rate of bleeding complication in patients with cirrhosis (10.9\% vs. $4.7 \% ; p=0.003$ ). Among the bleeding cases, 32 patients (66.7\%) had mild post-ERCP bleeding; 16 patients (33.3\%) had moderate post-ERCP bleeding (Table 2). Most bleeding events ( $\mathrm{n}=$ $32,66.7 \%$ ) were mild oozing that occurred during the proce- dure. No cases of massive bleeding occurred. Thirty-two bleeding episodes were controlled with electrical cauterization with a needle knife and/or hemoclip. No cases needed embolization or surgical management. There was no event of mortality related to bleeding.

\section{Risk factors related to post-ERCP bleeding}

Univariate analysis showed liver cirrhosis to be a significant risk factor related to post-ERCP bleeding ( $p=0.003)$. Because

Table 1. Patient Baseline Characteristics

\begin{tabular}{|ccccc}
\hline \multicolumn{1}{c}{ Variables } & $\begin{array}{c}\text { Total } \\
(\mathbf{n = 7 6 8 )}\end{array}$ & $\begin{array}{c}\text { Cirrhotic } \\
(\mathbf{n = 1 9 2 )}\end{array}$ & $\begin{array}{c}\text { Non-cirrhotic } \\
(\mathbf{n = 5 7 6 )}\end{array}$ & $\begin{array}{c}\boldsymbol{p} \\
\text { value }\end{array}$ \\
\hline Patient related variables & & & & \\
\hline Male & $592(77.1)$ & $148(77.1)$ & $444(77.1)$ & 1.000 \\
\hline Age (yr) & $59.4 \pm 9.5$ & $59.4 \pm 9.5$ & $59.4 \pm 9.5$ & 1.000 \\
\hline Body mass index & $22.6 \pm 3.5$ & $22.6 \pm 3.5$ & $22.7 \pm 3.5$ & 0.702 \\
\hline Prothrombin time (INR) & $1.01 \pm 0.21$ & $1.08 \pm 0.23$ & $0.98 \pm 0.20$ & 0.001 \\
\hline Platelet $\left.\times \times 10^{3} / \mu L\right)$ & $210.8 \pm 96.8$ & $147.4 \pm 77.4$ & $231.9 \pm 93.3$ & 0.001 \\
\hline Cardiovascular disease & $52(6.8)$ & $11(5.7)$ & $41(7.1)$ & 0.619 \\
\hline CKD & $29(3.8)$ & $17(8.9)$ & $12(2.1)$ & 0.001 \\
\hline Anticoagulant drug & $89(11.6)$ & $27(14.1)$ & $62(10.8)$ & 0.241 \\
\hline Procedure related variables & & & & \\
\hline Indication of ERCP & & & & \\
\hline CBD stone & $296(38.5)$ & $71(37.0)$ & $225(39.1)$ & 0.669 \\
\hline Bile duct obstruction & $335(43.6)$ & $98(51.0)$ & $237(41.1)$ & 0.019 \\
\hline Pancreatitis & $32(4.2)$ & $3(1.6)$ & $29(5.0)$ & 0.037 \\
\hline Procedure type & & & & \\
\hline Stent insertion ERBD & $250(32.6)$ & $63(32.8)$ & $187(32.5)$ & 0.929 \\
\hline EST & $350(45.6)$ & $93(48.4)$ & $257(44.6)$ & 0.359 \\
\hline Ballooning (EPBD) & $52(6.8)$ & $14(7.3)$ & $38(6.6)$ & 0.741 \\
\hline
\end{tabular}

ERCP, endoscopic retrograde cholangiopancreatography; ERBD, endoscopic retrograde biliary drainage; EST, endoscopic sphincterotomy; EPBD, endoscopic papillary balloon dilation; INR, international normalized ratio; CKD, chronic kidney disease; CBD, common bile duct.

Variables are expressed as mean \pm standard deviation or $\mathrm{n}(\%)$.

Table 2. Comparison of Post-ERCP Bleeding between Patients with Cirrhosis and Those without Cirrhosis

\begin{tabular}{lcccc}
\hline \multicolumn{1}{c}{ Adverse event } & $\begin{array}{c}\text { Total } \\
(\mathbf{n = 7 6 8 )}\end{array}$ & $\begin{array}{c}\text { Cirrhotic } \\
(\mathbf{n = 1 9 2 )}\end{array}$ & $\begin{array}{c}\text { Non-cirrhotic } \\
(\mathbf{n}=\mathbf{5 7 6 )}\end{array}$ & $\boldsymbol{p}$ value \\
\hline $\begin{array}{l}\text { Bleeding } \\
\text { Severity }\end{array}$ & $48(6.3)$ & $21(10.9)$ & $27(4.7)$ & 0.003 \\
$\quad$ Mild & $32(66.7)$ & $13(61.9)$ & $19(70.4)$ & 0.555 \\
$\quad$ Moderate & $16(33.3)$ & $8(38.1)$ & $8(29.6)$ & \\
$\quad$ Severe & $0(0.0)$ & $0(0.0)$ & $0(0.0)$ & \\
Time of bleeding & & & & 0.712 \\
$\quad$ Immediate & $39(81.3)$ & $18(85.7)$ & $21(77.8)$ & \\
$\quad$ Delayed & $9(18.8)$ & $3(14.3)$ & $6(22.2)$ & \\
Treatment modality & & & & 0.758 \\
$\quad$ Conservative & $16(33.3)$ & $6(28.6)$ & $10(37.0)$ & \\
$\quad$ Endoscopic & $32(66.7)$ & $15(71.4)$ & $17(63.0)$ & \\
\hline
\end{tabular}

ERCP, endoscopic retrograde cholangiopancreatography.

Variables are expressed as $n(\%)$. 
cirrhosis associated coagulopathy may increase the risk of procedure-related bleeding, we performed additional multivariate analysis. Liver cirrhosis was identified as an independent risk factor related to post-ERCP bleeding after adjustment for prothrombin time and duration of ERCP $(p=0.003)$ (Table 3$)$. Procedure types were not independent risk factors for postERCP bleeding.

\section{Bleeding complications according to the $\mathrm{CP}$ classification}

In subgroup analysis, there was a significant difference in the rate of post-procedure bleeding among patients with different CP classifications (Table 4). In multivariate analysis, CP class $\mathrm{C}$ was found to be associated with an increased incidence of post-ERCP bleeding in patients with cirrhosis (odds ratio 6.144, $95 \%$ confidence interval $1.320-28.606 ; p=0.021$ ).

\section{Complications according to procedure type}

The patients underwent various therapeutic maneuvers dur-

Table 3. Univariate and Multivariate Analysis of Risk Factors for PostERCP Bleeding

\begin{tabular}{lccccc}
\hline \multirow{2}{*}{ Variables } & Univariate & & \multicolumn{3}{c}{ Multivariate } \\
\cline { 2 - 2 } \cline { 5 - 6 } & p value & & Adjusted OR & $\mathbf{9 5 \%} \mathbf{~ C I}$ & $\boldsymbol{p}$ value \\
\hline Liver cirrhosis & 0.003 & & 2.497 & $1.377-4.530$ & 0.003 \\
$\begin{array}{l}\text { Prothrombin time } \\
\text { (INR) }\end{array}$ & 0.800 & & 1.198 & $0.298-4.819$ & 0.799 \\
EST & 0.871 & & & \\
EPBD & 0.316 & & & \\
Antiplatelet/ & 0.399 & & & \\
coagulant & 0.158 & 1.013 & $0.997-1.029$ & 0.103 \\
Duration of ERCP & & & & \\
Stent insertion & 0.293 & & & \\
\hline
\end{tabular}

ERCP, endoscopic retrograde cholangiopancreatography; $\mathrm{OR}$, odds ratio; $\mathrm{Cl}$, confidence interval; INR, international normalized ratio; EST, endoscopic sphincterotomy; EPBD, endoscopic papillary balloon dilatation.

Table 4. Multivariate Analysis of Risk Factors for Post-ERCP Bleeding in Cirrhotic Patients

\begin{tabular}{|c|c|c|c|}
\hline \multirow{2}{*}{ Variables } & \multicolumn{3}{|c|}{ Multivariate } \\
\hline & Adjusted OR & $95 \% \mathrm{Cl}$ & $p$ value \\
\hline \multicolumn{4}{|l|}{ Child-Pugh class } \\
\hline$A$ & 1 & & \\
\hline B & 2.533 & $0.704-9.112$ & 0.155 \\
\hline C & 6.144 & 1.320-28.606 & 0.021 \\
\hline Albumin & 0.539 & $0.156-1.867$ & 0.330 \\
\hline Platelet & 0.987 & $0.976-0.999$ & 0.032 \\
\hline CKD & 4.757 & $0.525-43.109$ & 0.165 \\
\hline Anticoagulant drugs & 1.690 & $0.257-11.116$ & 0.585 \\
\hline EST & 1.760 & $0.533-5.817$ & 0.354 \\
\hline EPBD & 0.552 & $0.052-5.889$ & 0.623 \\
\hline
\end{tabular}

ERCP, endoscopic retrograde cholangiopancreatography; $\mathrm{OR}$, odds ratio; $\mathrm{Cl}$, confidence interval; CKD, chronic kidney disease; EST, endoscopic sphincterotomy; EPBD, endoscopic papillary balloon dilatation. ing ERCP, which were individualized based on indications and intraprocedural findings. Among patients with liver cirrhosis, 93 patients (48.4\%) underwent EST; however, there was no significant difference in the rate of post-ERCP bleeding in patients who underwent biliary sphincterotomy, compared with those who did not have a sphincterotomy ( $10.8 \%$ vs. $11.1 \%, p=0.561)$ (Supplementary Table 1, only online). We further analyzed the events of PEP and cholangitis because a high frequency of adverse events, including PEP and cholangitis, occurred in patients with cirrhosis undergoing ERCP. In the present study, there were no significant differences in the occurrences of PEP and cholangitis ( $p=0.507$ and $p=0.489$ ) between the cirrhotic and non-cirrhotic groups (Supplementary Table 2, only online). In multivariate analysis, stent insertion was found to be associated with increased incidences of PEP and cholangitis ( $p=0.005$ and $p=0.029$ ) (Supplementary Tables 3 and 4, only online).

\section{DISCUSSION}

In this study, we analyzed our data after propensity score matching according to identified variables including sex and age. We confirmed that patients with liver cirrhosis face a greater risk of experiencing post-ERCP bleeding: liver cirrhosis was an independent risk factor associated with post-ERCP bleeding. These results were not changed after further adjustment for duration of ERCP, procedure type, and prothrombin time.

Patients with liver cirrhosis are predisposed to developing underlying coagulopathy. Because of poor hepatic synthetic function, these patients have coagulation disorders and low platelet counts, which are risk factors of post-procedure bleeding. Zhang, et al..$^{15}$ reported that post-ERCP bleeding was increased in patients with Model for End-stage Liver Disease (MELD) scores >11.5. Similar to previous studies, patients with $\mathrm{CP}$ class $\mathrm{C}$ cirrhosis experienced significantly more bleeding than those with $\mathrm{CP}$ class A or B in this study.

ERCP with sphincterotomy has been considered to pose a higher risk for post-procedure bleeding. ${ }^{4}$ In a previous study, complications of EST occur in about $10 \%$ of patients, and 2-3\% may have a prolonged hospital stay. ${ }^{20}$ The presence of varices, which can develop in the duodenum in patients with cirrhosis, may increase the risk of bleeding after sphincterotomy. However, in this study, bleeding risk after sphincterotomy did not show any statistically significant difference from the patients with cirrhosis, compared to those without cirrhosis. Regardless of the sphincterotomy, due to a tendency for patients with cirrhosis to bleed, bleeding complications of ERCP are considered to occur. Accordingly, the rate of bleeding was higher in patients with cirrhosis in this study.

Mariante-Neto, et al..$^{21}$ and Romano, et al..$^{22}$ reported that lower levels of creatinine may influence the low prognostic value of MELD score, which is associated post-ERCP bleed- 
ing, and suggested that sex may have an impact on the prognostic value of both MELD and CP scores. Herein, we matched all patients by age and sex. We analyzed the impact of liver cirrhosis on post-ERCP bleeding both without adjustment for platelet/prothrombin time and with it. After all of this, regardless of platelet/prothrombin time, liver cirrhosis was a significant risk factor in this study.

Our study has several limitations. First, the retrospective nature may lead to an inherent selection bias. To overcome this limitation, we performed propensity score matching. This study is based on a cohort from large representative tertiary referral hospitals. Further, we performed additional adjustment for platelet, albumin, and prothrombin time to reduce the selection bias. After matching, more than $50 \%$ of therapeutic ERCPs was performed in this study, and degree of cirrhosis was analyzed. Second, the definition of bleeding events has not been standardized in previous studies. ${ }^{11,13-15,23,24}$ The number of post-ERCP bleedings may depend on the definition of bleeding associated with a procedure. In this study, we categorized the bleeding grade according to the clinical and laboratory findings to find the effect of ERCP in patients with cirrhosis.

In conclusion, the incidence of post-ERCP bleeding in patients with liver cirrhosis is remarkably high after diagnostic and therapeutic ERCP. CP classification can be a valuable predictive factor for ERCP-related adverse events, especially in terms of bleeding. Bleeding problems may be related to the formation of a powerful collateral vascular bed in the area of the duodenum due to portal hypertension. In this regard, therapeutic ERCP in patients with liver cirrhosis should be used according to absolute indications and with high caution. For diagnostic purposes, minimally invasive methods, such as magnetic resonance cholangiopancreatography, endoscopic ultrasound, etc., may be more appropriate.

\section{ACKNOWLEDGEMENTS}

Pray for the bliss of dead of Ji Yeon Kim.

\section{AUTHOR CONTRIBUTIONS}

Ji Yeon Kim, Hee Seung Lee: data collection, data analysis, study conception, and manuscript preparation. Moon Jae Chung: critical revision of manuscript, data quality review. Jeong Youp Park: critical revision of manuscript, data quality review. Seung Woo Park: critical revision of manuscript, data quality review. Si Young Song: critical revision of manuscript, data quality review. Seungmin Bang: study conception, critical revision of manuscript, overall study supervision. All authors approved the final manuscript.

\section{ORCID iDs}

Hee Seung Lee Moon Jae Chung Jeong Youp Park Seung Woo Park https://orcid.org/0000-0002-2825-3160

https://orcid.org/0000-0002-5920-8549 https://orcid.org/0000-0003-0110-8606 https://orcid.org/0000-0001-8230-964X
Si Young Song

https://orcid.org/0000-0002-1417-4314

Seungmin Bang

https://orcid.org/0000-0001-5209-8351

\section{REFERENCES}

1. Adler DG, Baron TH, Davila RE, Egan J, Hirota WK, Leighton JA, et al. ASGE guideline: the role of ERCP in diseases of the biliary tract and the pancreas. Gastrointest Endosc 2005;62:1-8.

2. Sakai Y, Tsuyuguchi T, Sugiyama H, Nishikawa T, Tawada K, Saito $\mathrm{M}$, et al. Current situation of endoscopic treatment for common bile duct stones. Hepatogastroenterology 2012;59:1712-6.

3. Tulassay Z, Zagoni T, Kotrlik J. Complications of endoscopic biliary sphincterotomy. N Engl J Med 1997;336:963; author reply 963-4.

4. ASGE Standards of Practice Committee, Anderson MA, Fisher L, Jain R, Evans JA, Appalaneni V, Ben-Menachem T, et al. Complications of ERCP. Gastrointest Endosc 2012;75:467-73.

5. Andriulli A, Loperfido S, Napolitano G, Niro G, Valvano MR, Spirito $\mathrm{F}$, et al. Incidence rates of post-ERCP complications: a systematic survey of prospective studies. Am J Gastroenterol 2007;102: 1781-8.

6. Masci E, Toti G, Mariani A, Curioni S, Lomazzi A, Dinelli M, et al. Complications of diagnostic and therapeutic ERCP: a prospective multicenter study. Am J Gastroenterol 2001;96:417-23.

7. Loperfido S, Angelini G, Benedetti G, Chilovi F, Costan F, De Berardinis $\mathrm{F}$, et al. Major early complications from diagnostic and therapeutic ERCP: a prospective multicenter study. Gastrointest Endosc 1998;48:1-10.

8. Wilcox CM, Canakis J, Mönkemüller KE, Bondora AW, Geels W. Patterns of bleeding after endoscopic sphincterotomy, the subsequent risk of bleeding, and the role of epinephrine injection. Am J Gastroenterol 2004;99:244-8.

9. Nelson DB, Freeman ML. Major hemorrhage from endoscopic sphincterotomy: risk factor analysis. J Clin Gastroenterol 1994;19: 283-7.

10. Freeman ML, Nelson DB, Sherman S, Haber GB, Herman ME, Dorsher PJ, et al. Complications of endoscopic biliary sphincterotomy. N Engl J Med 1996;335:909-18.

11. Inamdar S, Berzin TM, Berkowitz J, Sejpal DV, Sawhney MS, Chutanni R, et al. Decompensated cirrhosis may be a risk factor for adverse events in endoscopic retrograde cholangiopancreatography. Liver Int 2016;36:1457-63.

12. Li DM, Zhao J, Zhao Q, Qin H, Wang B, Li RX, et al. Safety and efficacy of endoscopic retrograde cholangiopancreatography for common bile duct stones in liver cirrhotic patients. J Huazhong Univ Sci Technolog Med Sci 2014;34:612-5.

13. Lee MH, Tsou YK, Lin CH, Lee CS, Liu NJ, Sung KF, et al. Predictors of re-bleeding after endoscopic hemostasis for delayed post-endoscopic sphincterotomy bleeding. World J Gastroenterol 2016;22: 3196-201.

14. Adler DG, Haseeb A, Francis G, Kistler CA, Kaplan J, Ghumman SS, et al. Efficacy and safety of therapeutic ERCP in patients with cirrhosis: a large multicenter study. Gastrointest Endosc 2016;83: 353-9.

15. Zhang J, Ye L, Zhang J, Lin M, He S, Mao X, et al. MELD scores and Child-Pugh classifications predict the outcomes of ERCP in cirrhotic patients with choledocholithiasis: a retrospective cohort study. Medicine (Baltimore) 2015;94:e433.

16. Prat F, Tennenbaum R, Ponsot P, Altman C, Pelletier G, Fritsch J, et al. Endoscopic sphincterotomy in patients with liver cirrhosis. Gastrointest Endosc 1996;43(2 Pt 1):127-31.

17. Kawabe T, Komatsu Y, Tada M, Toda N, Ohashi M, Shiratori Y, et al. Endoscopic papillary balloon dilation in cirrhotic patients: removal of common bile duct stones without sphincterotomy. En- 
doscopy 1996;28:694-8.

18. Park DH, Kim MH, Lee SK, Lee SS, Choi JS, Song MH, et al. Endoscopic sphincterotomy vs. endoscopic papillary balloon dilation for choledocholithiasis in patients with liver cirrhosis and coagulopathy. Gastrointest Endosc 2004;60:180-5.

19. Kim DY, Kim SU, Ahn SH, Park JY, Lee JM, Park YN, et al. Usefulness of FibroScan for detection of early compensated liver cirrhosis in chronic hepatitis B. Dig Dis Sci 2009;54:1758-63.

20. Cotton PB, Lehman G, Vennes J, Geenen JE, Russell RC, Meyers WC, et al. Endoscopic sphincterotomy complications and their management: an attempt at consensus. Gastrointest Endosc 1991; 37:383-93.

21. Mariante-Neto G, Marroni CP, Fleck Junior AM, Marroni CA, Zan- otelli ML, Cantisani G, et al. Impact of creatinine values on MELD scores in male and female candidates for liver transplantation. Ann Hepatol 2013;12:434-9.

22. Romano TG, Schmidtbauer I, Silva FM, Pompilio CE, D’Albuquerque LA, Macedo E. Role of MELD score and serum creatinine as prognostic tools for the development of acute kidney injury after liver transplantation. PLoS One 2013;8:e64089.

23. Navaneethan U, Njei B, Zhu X, Kommaraju K, Parsi MA, Varadarajulu S. Safety of ERCP in patients with liver cirrhosis: a national database study. Endosc Int Open 2017;5:E303-14.

24. Kim KO, Kim TN, Kim SB, Lee JY. Characteristics of delayed hemorrhage after endoscopic sphincterotomy. J Gastroenterol Hepatol 2010;25:532-8. 